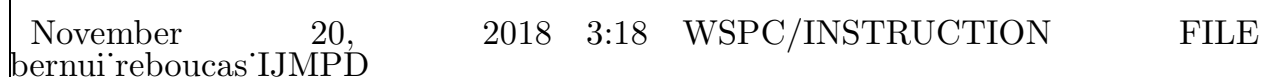

International Journal of Modern Physics D

(C) World Scientific Publishing Company

\title{
A STUDY OF GAUSSIANITY IN CMB BAND MAPS
}

\author{
ARMANDO BERNUI ${ }^{1}$ \\ ${ }^{1}$ Instituto de Ciências Exatas, \\ Universidade Federal de Itajubá, 37500-903, Itajubá - MG, Brazil \\ abernui@gmail.com \\ MARCELO J. REBOUÇAS ${ }^{2}$ and ANTONIO F. F. TEIXEIRA ${ }^{2}$ \\ ${ }^{2}$ Centro Brasileiro de Pesquisas Físicas, \\ 22290-180, Rio de Janeiro - RJ, Brazil \\ reboucas@cbpf.br ; teixeira@cbpf.br
}

\begin{abstract}
The detection of non-Gaussianity in the CMB data would rule out a number of inflationary models. A null detection of non-Gaussianity, instead, would exclude alternative models for the early universe. Thus, a detection or non-detection of primordial nonGaussianity in the CMB data is crucial to discriminate among inflationary models, and to test alternative scenarios. However, there are various non-cosmological sources of non-Gaussianity. This makes important to employ different indicators in order to detect distinct forms of non-Gaussianity in CMB data. Recently, we proposed two new indicators to measure deviation from Gaussianity on large angular scales, and used them to study the Gaussianity of the raw band WMAP maps with and without the KQ75 mask. Here we extend this work by using these indicators to perform similar analyses of deviation from Gaussianity of the foreground-reduced $\mathrm{Q}, \mathrm{V}$, and $\mathrm{W}$ band maps. We show that there is a significant deviation from Gaussianity in the considered full-sky maps, which is reduced to a level consistent with Gaussianity when the KQ75 mask is employed.
\end{abstract}

Keywords: Cosmic microwave background; non-Gaussianity; physics of the early universe.

\section{Introduction}

Cosmic Microwave Background (CMB) data from the Wilkinson Microwave Anisotropy Probe (WMAP $)^{1}$ is under rigorous scrutiny for the possible deviations from Gaussianity $\sqrt{2}$ and for statistical isotropy $\sqrt{5}$ in the CMB temperature field. A detection or non-detection of primordial non-Gaussianity in the CMB data is crucial to discriminate among inflationary models, and to test alternative scenarios. ${ }^{6}$ However, since non-cosmological effects can produce non-Gaussianity in the CMB data, the extraction of a possible primordial non-Gaussianity is not a simple enterprise. Besides, one does not expect that any single statistical estimator can be sensitive to all possible forms of non-Gaussianity. It is therefore important to test CMB data for deviations from Gaussianity by using different statistical tools, since they can potentially provide information about multiple forms of non-Gaussianity that may be present in CMB data. In a previous work ${ }^{3}$ we introduced two indicators, based 
on skewness and kurtosis of CMB data, and used them to study the deviation from Gaussianity in the WMAP raw band (uncleaned) maps. In the present work, we complement this study of WMAP five-year data by performing a similar analysis but now using the foreground-reduced $\mathrm{Q}, \mathrm{V}$, and $\mathrm{W}$ band maps with and without the KQ75 mask. We show that there is a significant deviation from Gaussianity in these foreground-reduced full-sky band maps, which is reduced to a level that is consistent with Gaussianity when the KQ75 mask is used.

\section{Non-Gaussian indicators}

In this section we briefly present how to construct our non-Gaussinity indicators. For a detailed discussion of these indicators we refer the readers to Ref. 3 and Ref. 4 ,

Consider a discrete set of points $\left\{j=1, \ldots, N_{\mathrm{c}}\right\}$ uniformly distributed on the sphere $S^{2}$ as the center of spherical caps of a given aperture $\gamma$, and calculate for each cap $j$ the real numbers

$$
S_{j} \equiv \frac{1}{N_{\mathrm{p}} \sigma_{j}^{3}} \sum_{i=1}^{N_{\mathrm{p}}}\left(T_{i}-\bar{T}_{j}\right)^{3}, \quad K_{j} \equiv \frac{1}{N_{\mathrm{p}} \sigma_{j}^{4}} \sum_{i=1}^{N_{\mathrm{p}}}\left(T_{i}-\bar{T}_{j}\right)^{4}-3,
$$

where $N_{\mathrm{p}}$ is the number of pixels in the $j^{\text {th }}$ cap, $T_{i}$ is the temperature at the $i^{\text {th }}$ pixel, $\bar{T}_{j}$ is the CMB mean temperature on the $j^{\text {th }}$ cap, and $\sigma_{j}$ is the standard deviation for each $j$. The sets of values $\left\{S_{j}, j=1, \ldots, N_{\mathrm{c}}\right\}$ and $\left\{K_{j}, j=1, \ldots, N_{\mathrm{c}}\right\}$ can then be viewed as measures of the non-Gaussianity in the directions $\left(\theta_{j}, \phi_{j}\right)$ of the center of the $j^{\text {th }}$ cap. Patching together the $\left\{S_{j}\right\}$ and $\left\{K_{j}\right\}$ values, we obtain the skewness $S=S(\theta, \phi)$ and kurtosis $K=K(\theta, \phi)$ functions defined on $S^{2}$. Throughout this work the Mollweide projection of the functions $S=S(\theta, \phi)$ and $K=K(\theta, \phi)$ are named, respectively, $S$-map and $K$-map. Clearly, one can expand each of these functions in their spherical harmonics and calculate their angular power spectrum. For the skewness function $S=S(\theta, \phi)=\left\{S_{j}, j=1, \ldots, N_{\mathrm{c}}\right\}$, for example, one has

$$
S(\theta, \phi)=\sum_{\ell=0}^{\infty} \sum_{m=-\ell}^{\ell} b_{\ell m} Y_{\ell m}(\theta, \phi) \Longrightarrow S_{\ell}=\frac{1}{2 \ell+1} \sum_{m}\left|b_{\ell m}\right|^{2},
$$

where $S_{\ell}$ is the corresponding angular power spectrum. Similar expressions hold for the $K=K(\theta, \phi)$.

To obtain quantitative information for each multipole component $\ell$ about the non-Gaussianity at large-angles (low $\ell$ ) from the $S$ and $K$ maps (obtained from the band maps) we calculate the power spectra $S_{\ell}$ and $K_{\ell}$. Then, to estimate the statistical significance of each multipole, we compare $S_{\ell}$ and $K_{\ell}$ to the mean multipole values $\bar{S}_{\ell}$ and $\bar{K}_{\ell}$ calculated by averaging over 1000 power spectra of $S$ and $K$ maps obtained from Monte Carlo (MC) Gaussian CMB maps

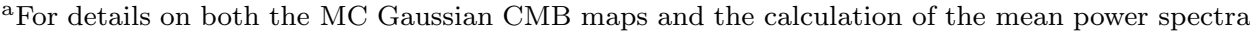
$\bar{S}_{\ell}$ and $\bar{K}_{\ell}$ see Refs. 3 and 4 . 


\section{Results and Conclusions}

In this section we report the results of our Gaussianity analysis performed with the indicators discussed in the previous section.

To minimize the statistical noise, in the calculations of $S$ and $K$ maps from the foreground-reduced $\mathrm{Q}, \mathrm{V}, \mathrm{W}$ input maps, we have scanned the celestial sphere with spherical caps of aperture $\gamma=90^{\circ}$, whose centers are located at 12288 points homogeneously distributed on the celestial sphere. The input maps we have used in our analyses have the resolution HEALPix parameter $N_{\text {side }}=256,7$ which corresponds to a partition of the celestial sphere into 786432 pixels. The calculations of the $S$ and $K$ maps by scanning the CMB masked maps sometimes include caps whose center is within or close to the KQ75 masked region. In these cases, the calculations are made with a smaller number of pixels $N_{\mathrm{p}}$. Finally, we note that examples of $S$ and $K$ maps can be found in Refs. 3 and 4 .

In Fig. 1 we show the angular power spectra of the $S$ maps and $K$ maps calculated from the WMAP foreground-reduced Q, V, and W CMB band maps by considering the data in the full-sky and KQ75 masked maps. These angular power spectra show that the KQ75 mask lowers the non-Gaussianity to a level below $95.4 \%$ CL.

An overall quantitative measure of deviation from Gaussianity for $\ell=1-10$ is obtained through the $\chi^{2}$ goodness-of-fit test by calculating the $\chi^{2} /$ dof (where dof stands for degrees of freedom) of the spectra values $S_{\ell}$ and $K_{\ell}$ calculated from the Q, V, and W band maps as compared to the mean power spectra $\bar{S}_{\ell}$ and $\bar{K}_{\ell}$ obtained from $S$ and $K$ maps calculated from MC CMB maps. The results regarding the masked and the full-sky maps are shown in Table 1 . The values obtained in the masked maps analysis $\left(\chi^{2} \lesssim 10\right)$ indicate that these may be seen as consistent with Gaussianity, while the values for full-sky foreground-reduced maps $\left(\chi^{2}>10^{10}\right)$ shows a large deviation from Gaussianity.

Table 1. Values for the ratio $\chi^{2} /$ dof that measure the goodness-of-fit of the $S_{\ell}$ and $K_{\ell}$ spectra from the WMAP foreground-reduced band maps as compared to the mean spectra $\bar{S}_{\ell}$ and $\bar{K}_{\ell}$, respectively. The values for the full-sky and KQ75 masked maps are shown.

\begin{tabular}{|c|c|c|c|c|c|c|}
\hline$\chi^{2} \backslash \mathrm{CMB}$ maps & $\mathrm{Q}$ [full-sky] & $\mathrm{V}$ [full-sky] & $\mathrm{W}$ [full-sky] & $\mathrm{Q}\left[K Q^{7} 75\right]$ & $\mathrm{V}\left[K Q^{75}\right]$ & $\mathrm{W}\left[K Q^{75}\right]$ \\
\hline$S_{\ell}$ & $9.4 \times 10^{11}$ & $1.2 \times 10^{12}$ & $7.3 \times 10^{10}$ & 3.1 & 4.4 & 3.4 \\
\hline$K_{\ell}$ & $1.2 \times 10^{21}$ & $3.3 \times 10^{21}$ & $1.0 \times 10^{20}$ & 6.3 & 6.5 & 6.0 \\
\hline
\end{tabular}

\section{Acknowledgments}

This work is supported by Conselho Nacional de Desenvolvimento Científico e Tecnológico (CNPq) - Brasil, under grant No. 472436/2007-4. A.B. and M.J.R. thank $\mathrm{CNPq}$ for their grants. We acknowledge the use of the Legacy Archive for Mi- 

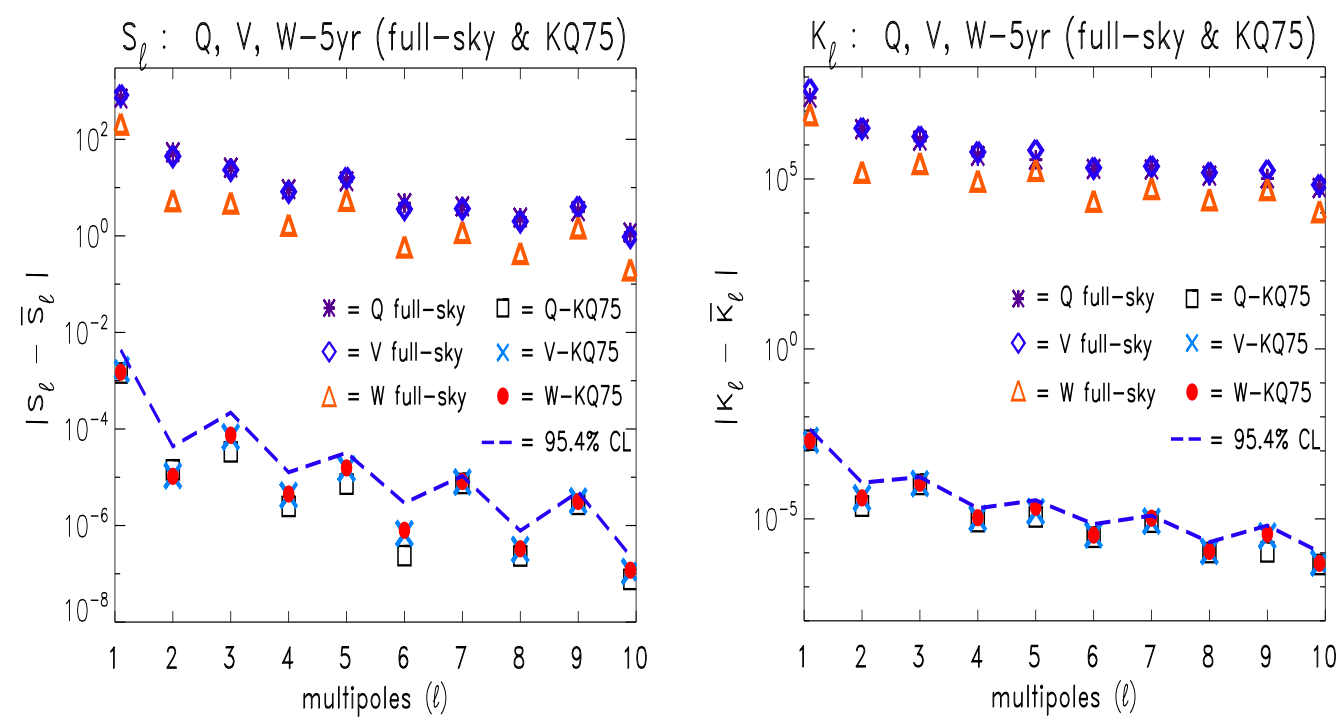

Fig. 1. Low $\ell$ differential power spectra of skewness $\left|S_{\ell}-\bar{S}_{\ell}\right|$ (left) and kurtosis (right) $\left|K_{\ell}-\bar{K}_{\ell}\right|$ calculated from the WMAP foreground-reduced band maps. The values for the full-sky and KQ75 masked maps are shown. The $95.4 \%$ confidence level (obtained from $S$ and $K$ maps calculated from MC Gaussian CMB maps) is indicated by the dashed line. More details in the text.

crowave Background Data Analysis (LAMBDA) 1 Some of the results in this paper were derived using the HEALPix package! 7

\section{References}

1. G. Hinshaw et al., Astrophys. J. Suppl. Ser. 180 (2009) 225.

2. C. Monteserín et al., Mon. Not. R. Astron. Soc. 387 (2008) 209; B. Lew, JCAP 08 (2008) 017; A. Bernui, C. Tsallis and T. Villela, Europhys. Lett. 78 (2007) 19001; Phys. Lett. A 356 (2006) 426; Y. Wiaux et al., Mon. Not. Roy. Astron. Soc. 385 (2008) 939; P. D. Naselsky et al., arXiv:0712.1118 [astro-ph]; J. D. McEwen et al., Mon. Not. R. Astron. Soc. 388 (2008) 659; P. Vielva and J. L. Sanz, Mon. Not. R. Astron. Soc. 397 (2009) 837; G. Rossmanith, C. Räth, A. J. Banday and G. Morfill, arXiv:0905.2854 [astro-ph.CO]; M. Kawasaki et al., JCAP 11 (2008) 019; M. Kawasaki, K. Nakayama and F. Takahashi, JCAP 01 (2009) 026; M. Kawasaki et al., JCAP 01 (2009) 042; D. Pietrobon et al., Mon. Not. Roy. Astron. Soc. 396 (2009) 1682; D. Pietrobon et al., Mon. Not. Roy. Astron. Soc. 402 (2010) L34; A. Bernui and M. J. Rebouças, Int. J. Mod. Phys. A 24 (2009) 1664; M. Cruz, E. Martínez-González and P. Vielva, arXiv:0901.1986 [astro-ph]; Y. Ayaita, M. Weber and C. Wetterich, Phys. Rev. D 81 (2010) 023507; P. Cabella et al., arXiv:0910.4362 [astro-ph.CO].

3. A. Bernui and M. J. Rebouças, Phys. Rev. D 79 (2009) 063528.

4. A. Bernui and M. J. Rebouças, Phys. Rev. D 81 (2010) 063533.

5. P. Vielva, Y. Wiaux, E. Martínez-González and P. Vandergheynst, Mon. Not. Roy. Astron. Soc. 381 (2007) 932; A. Bernui et al., Astron. Astrophys. 454 (2006) 409; T. Ghosh, A. Hajian and T. Souradeep, Phys. Rev. D 75 (2007) 083007; A. Gruppuso, Phys. Rev. D 76 (2007) 083010; A. Bernui, B. Mota, M. J. Rebouças and R. Tavakol, 
November $\quad 20, \quad 2018 \quad 3: 18 \quad$ WSPC/INSTRUCTION $\quad$ FILE

Astron. Astrophys. 464 (2007) 479; A. Bernui, B. Mota, M. J. Rebouças and R. Tavakol, Int. J. Mod. Phys. D 16 (2007) 411; B. Lew, JCAP 09 (2008) 023; P. K. Samal, R. Saha, P. Jain and J. P. Ralston, Mon. Not. R. Astron. Soc. 385 (2008) 1718; T. Koivisto and D. F. Mota, JCAP 06 (2008) 018; P. K. Samal, R. Saha, P. Jain and J. P. Ralston, Mon. Not. R. Astron. Soc. 396 (2009) 511; C. M. Hirata, JCAP 09 (2009) 011; C. Caprini, F. Finelli, D. Paoletti and A. Riotto, JCAP 06 (2009) 021; J. Kim and P. Naselsky, JCAP 07 (2009) 041; T. Kahniashvili, G. Lavrelashvili and B. Ratra, Phys. Rev. D 78 (2008) 063012; A. Bernui and W. S. Hipólito-Ricaldi, Mon. Not. R. Astron. Soc. 389 (2008) 1453; I. Y. Areféva, N. V. Bulatov, L. V. Joukovskaya and S. Y. Vernov, Phys. Rev. D 80 (2009) 083532; I. Y. Areféva, N. V. Bulatov and S. Y. Vernov, arXiv:0911.5105 [hep-th]; A. Bernui, Phys. Rev. D 78 (2008) 063531; A. Bernui, Phys. Rev. D 80 (2009) 123010; Y. Shtanov and H. Pyatkovska, Phys. Rev. D 80 (2009) 023521; G. P. Holder, K. M. Nollett and A. van Engelen, arXiv:0907.3919 [astro-ph.CO]; L. R. Abramo et al., Phys. Rev. D 74 (2006) 063506; L. R. Abramo, A. Bernui and T. S. Pereira, JCAP 12 (2009) 013; A. L. Erickcek, C. M. Hirata and M. Kamionkowski, Phys. Rev. D 80 (2009) 083507; J. F. Donoghue, K. Dutta and A. Ross, Phys. Rev. D 80 (2009) 023526; N. Joshi, S. Jhingan, T. Souradeep and A. Hajian, arXiv:0912.3217 [astro-ph.CO]; T. R. Seshadri and K. Subramanian, Phys. Rev. Lett. 103 (2009) 081303; T.-P. Li, H. Liu, L.-M. Song, S.-L. Xiong and J.-Y. Nie, arXiv:0905.0075 [astro-ph.CO]; M. Frommert and T. A. Ensslin, arXiv:0908.0453 [astro-ph.CO]; H.-C. Kim and M. Minamitsuji, arXiv:1002.1361 [gr-qc]; A. Gruppuso and K. M. Górski, JCAP 03 (2010) 019; D. G. Yamazaki, K. Ichiki, T. Kajino and G. J. Mathews, Phys. Rev. D 81 (2010) 023008; L. R. Abramo and T. S. Pereira, arXiv:1002.3173 [astro-ph.CO]; A. R. Pullen and C. M. Hirata, arXiv:1003.0673 [astroph.CO]; F. Paci, A. Gruppuso, F. Finelli, P. Cabella, A. De Rosa, N. Mandolesi and P. Natoli, arXiv:1002.4745 [astro-ph.CO].

6. N. Bartolo et al., Phys. Rep. 402 (2004) 103; E. Komatsu et al., arXiv:0902.4759.

7. K. M. Górski et al., Astrophys. J. 622 (2005) 759. 